\title{
miR-4295 promotes cell proliferation, migration and invasion of osteosarcoma through targeting interferon regulatory factor 1
}

\author{
JIN PEI CHENG, BIN HUANG, JUN HU DUAN, KAI JUN YI and ZHENG LING ZHUANG \\ Department of Orthopaedics, Xiangyang No. 1 People's Hospital, \\ Hubei University of Medicine, Xiangyang, Hubei 441000, P.R. China
}

Received March 30, 2019; Accepted July 23, 2020

DOI: $10.3892 / \mathrm{ol} .2020 .12123$

\begin{abstract}
Osteosarcoma (OS) is the most common form of primary malignant bone tumor. Despite encouraging progress in the treatment of OS, the survival rate for patients with OS has remained unchanged over the past 40 years. It has been established that miRNA plays a crucial regulatory role in the progression and development of OS. To explore the potential association of miRNAs with OS, bioinformatics techniques were used to screen for differentially expressed miRNA genes in OS in the Gene Expression Omnibus database. In the GSE70367 database, it was revealed that miR-4295 expression was abnormally elevated in the expression of OS cells. To characterize the potential function of miR-4295 in OS, the expression levels of miR-4295 in 30 samples of OS and adjacent normal tissues was examined. The results revealed that the expression of miR-4295 was significantly increased in OS tissues compared with the paired normal tissues. Moreover, the expression levels of miR-4295 in OS cell lines (MG-63 and Saos-2) were significantly higher compared with those in the normal human mesenchymal stem cells. In addition, miR-4295 was associated with OS cell proliferation, migration and invasion. Furthermore, it was demonstrated that the expression of interferon regulatory factor (IRF)1, a tumor suppressor, was regulated by miR-4295 directly in OS cells. Taken together, the present results revealed that miR-4295 may act as a tumor activator by targeting IRF1 during the progression of OS. Investigating miR-4295 may provide novel insight into the mechanisms of OS metastasis, and inhibition and targeting miR-4295 may be a novel therapeutic strategy for the treatment of OS.
\end{abstract}

Correspondence to: Dr Zheng Ling Zhuang, Department of Orthopaedics, Xiangyang No. 1 People's Hospital, Hubei University of Medicine, 15 Jiefang Road, Xiangyang, Hubei 441000, P.R. China E-mail: zhuang-zl@163.com

Key words: miR-4295, osteosarcoma, interferon regulatory factor 1, proliferation, migration, invasion

\section{Introduction}

Osteosarcoma (OS) is the most frequent form of primary malignant bone tumor and it mainly affects children, adolescents or young adults (1). The peak incidence of the disease is in patients aged 15-25 years old, and is more commonly observed in males compared with females (1). OS exhibits a high degree of malignancy and tends to metastasize early, with a clinical metastasis rate of $\sim 20 \%$, with lung metastases being the most common (2-4). The course of OS progresses rapidly, which is life threatening and has a high mortality rate (5). The prognosis for OS distant metastasis cases remains poor (5). The mechanisms underlying the pathogenesis of OS remains unclear. Studies have reported that a variety of oncogenes and tumor suppressor genes have significant contributions in the development of OS, but the specific molecular mechanisms remain unclear (6-9). Therefore, a better understanding of the mechanisms underpinning the development of OS may provide an important theoretical basis for the clinical treatment of OS.

MicroRNAs (miRNAs/miRs), small non-coding RNAs of 20-25 nucleotides in length, were first discovered by Lee et al in 1993 in Caenorhabditis elegans. Gene expression can be regulated by miRNAs after transcription, regulating protein abundance by promoting mRNA degradation or translational inhibition, thereby acting as an oncogene or tumor suppressor $(10,11)$. Studies have revealed that miRNAs play an important role in various biological processes, such as cell differentiation, proliferation, apoptosis, migration, metabolism and defense (12-14). In OS, a variety of abnormally expressed miRNAs had been identified, which are involved in biological processes, including invasion and metastasis, and affect the malignant biological behavior of OS cells by regulating different target genes, such as AKT, TRAF3 and p57 $(15,16)$. However, it is still unclear which specific miRNAs specifically regulate the development and progression of OS.

Recently, several studies have shown that miR-4295 may act as an oncogene and a potential biomarker for cancer diagnosis and treatment $(17,18)$. It was reported that miR-4295 can inhibit the $\mathrm{G}_{0} / \mathrm{G}_{1}$ arrest and apoptosis of glioma cells and promote cell proliferation and cell activity in glioma cells (19). High expression of miR-4295 may contribute to proliferation and invasion of pancreatic ductal adenocarcinoma (20). Taken together, it was hypothesized that miR-4295 may serve an important role in the development of OS. 
Interferon regulatory factor 1 (IRF1) is a member of the interferon family (IRFs) and widely expressed in various tissues (21). IRFs are a family of transcription factors that regulate interferon expression, antivirus and antitumor activity. The IRFI gene has been studied in multiple species (22) and IRF1 selectively modulates different sets of genes, such as p53, p21 and TRAIL, and contributed to the cellular immune response (23). Several malignant blood system diseases, such as acute leukemia and myelodysplastic syndrome, and a variety of cancer types, were accompanied by abnormal expression of the IRF1 gene $(22,24)$.

The present study aimed to explore the potential function of miR-4295 in OS and examine the influence of the miR-4295 target genes.

\section{Materials and methods}

Tissue specimens and lentivirus All tissue specimens obtained after surgery were immediately frozen using liquid nitrogen and then stored at $-80^{\circ} \mathrm{C}$ until use. No patients received any treatment, including chemotherapy or radiation therapy, before surgery. A total of 15 tumor tissues and adjacent normal tissues ( $>3 \mathrm{~cm}$ away from the tumor tissues) were collected from 10 men and 5 women (age range, 10-65 years; median age, 32 years). Amputation was performed in 9 cases whereas local resection of mass was performed in 6 cases. This study was based on the latest revised principles outlined in the Helsinki Declaration. The acquisition and analysis of the OS cancer specimens was approved by the Ethics Committee of Xiangyang No. 1 People's Hospital and the patients provided written informed consent. Lentivirus used in the present study was provided by Shanghai GenePharma, Co., Ltd. LV-vector lentivirus was the negative control. LV-IRF1 corresponds to the IRF1 expression group.

Cell culture. Human OS cell lines (MG-63 and Saos-2) and normal human mesenchymal stem cells (hMSC) were purchased from The Cell Bank of Type Culture Collection of the Chinese Academy of Sciences. Cells were cultured in Dulbecco's modified Eagle's medium (DMEM) with $10 \%$ fetal bovine serum (FBS) (Gibco; Thermo Fisher Scientific, Inc.) and incubated at $37^{\circ} \mathrm{C}$ with $5 \% \mathrm{CO}_{2}$.

$R N A$ isolation and reverse transcription-quantitative (RT-q) $P C R$. Total RNA was extracted from OS cells using TRIzol ${ }^{\circledR}$ (Thermo Fisher Scientifi, Inc.) and was reverse transcribed into cDNA using the 5xPrimeScript RT Master mix (Takara Bio, Inc.) according to the manufacturer's protocols. qPCR was performed using the 2xT5 Fast qPCR mix (SYBR Green) (TsingKe Biological Technology) with a LightCycler ${ }^{\circledR} 96$ Real-Time qPCR Detection system (Roche Diagnostics). RT-qPCR reactions were performed as follows: 40 cycles, denaturation, $94^{\circ} \mathrm{C}$ for $15 \mathrm{sec}$; annealing, extension at $62^{\circ} \mathrm{C}$ for $30 \mathrm{sec}$. GAPDH or U6 was used as a reference gene. The relative mRNA and miRNA expressions were calculated using the $2^{-\Delta \Delta \mathrm{Cq}}$ method (25). The primers used were as follows: GAPDH, Forward: 5'-ATCACCATCTTCCAGGAGGGA-3' and reverse: 5'-CCTTCTCCATGGTGGTGAAGAC-3'; U6, forward: 5'-CTCGCTTCGGCAGCACA-3' and reverse: 5'-AACGCTTCACGAATTTGCGT-3'; IRF1, forward: 5'-CTG
TGCGAGTGTACCGGATG-3' and reverse: 5'-ATCCCCACA TGACTTCCTCTT-3'; miR-4295 RT: 5'-GTCGTATCCAGT GCGTGTCGTGGAGTCGGCAATTGCACTGGATACGAC AAGGAA-3'; PCR, forward: 5'-GGGCAGUGCAAUGUU-3' and reverse: 5'-CAGTGCGTGTCGTGGAGT-3'. All results were derived from three independent amplifications.

Cell transfection. Cells $\left(3 \times 10^{5}\right)$ were seeded in six-well dishes, and transfected transiently with 200 pmol miR-4295 mimic/inhibitor/mimic non-targeting, scrambled/inhibitor non-targeting, scrambled (NC; Shanghai GenePharma, Co., Ltd.) using Lipofectamine ${ }^{\circledR} 2000$ (Thermo Fisher Scientific, Inc.) in accordance with the manufacturer's protocol. During transfection, DMEM without FBS was used. Cells were harvested after $48 \mathrm{~h}$ post transfection for RT-qPCR and the dual luciferase assay.

Cell proliferation assay. Cell proliferation was determined using a Cell Counting Kit (CCK)-8 assay (Dojindo Molecular Technologies, Inc.) in accordance with the manufacturer's protocol. To conduct this experiment, $5 \times 10^{3}$ cells were seeded in 96-well dishes and cultured in $100 \mu \mathrm{l}$ DMEM medium at $37^{\circ} \mathrm{C}$. After $12 \mathrm{~h}, 10 \mu \mathrm{lCCK}-8$ solution was added to each well and incubated for $1 \mathrm{~h}$ at $37^{\circ} \mathrm{C}$. Absorbance was then measured with a multifunctional microplate reader at $450 \mathrm{~nm}$. The assays were independently repeated $\geq 3$ times.

Cell migration and invasion assay. The migration and invasion ability of MG-63 and Saos-2 cells were evaluated using a Transwell chamber (Corning, Inc.). In total, $1 \times 10^{4}$ cells were seeded in the upper chamber without FBS. Then, $600 \mu \mathrm{l}$ DMEM containing $50 \mathrm{ml} / \mathrm{l} \mathrm{FBS}$ was added to the lower chamber and incubated for $24 \mathrm{~h}$ at $37^{\circ} \mathrm{C}$. The chamber was fixed with $4 \%$ paraformaldehyde at room temperature for $20 \mathrm{~min}$, washed with PBS three times, and stained with $0.1 \%$ crystal violet (Beyotime Institute of Biotechnology) for $15 \mathrm{~min}$ at room temperature. The unmigrated cells were wiped off gently by using cotton swabs. The migration ability of the cells was determined by counting manually the number of transmembrane cells. At least three fields of view were randomly observed in each group using light microscopy (magnification, x100).

The invasion ability of the cells was assayed in a Transwell chamber precoated with Matrigel (Corning Inc.). In total, $50 \mu 1$ of Matrigel collagen was diluted (Corning, Inc.) with $400 \mu \mathrm{l}$ of FBS-free DMEM medium (ice operation). Then, $60 \mu 1$ of the dilution was added to the upper chamber of the Transwell chamber and incubated for $4 \mathrm{~h}$ at $37^{\circ} \mathrm{C}$. The subsequent steps are the same as the migration assay. The invasive ability of the cells was determined by manually counting the number of transmembrane cells. At least three fields of view were randomly observed in each group by using Leica DMi8 microscope (Leica Microsystems, Inc.; magnification, x100).

Wound healing assay. The cells were plated in a 6-well plate one day in advance. A $20 \mu \mathrm{l}$ pipette tip was used to create a scratch wound across the cells on the plate, and then the plate was washed twice with PBS to remove any floating cells. Low serum medium containing $2 \%$ FBS was replaced after scraping. Images of the cells migrated across the wounds were 
A

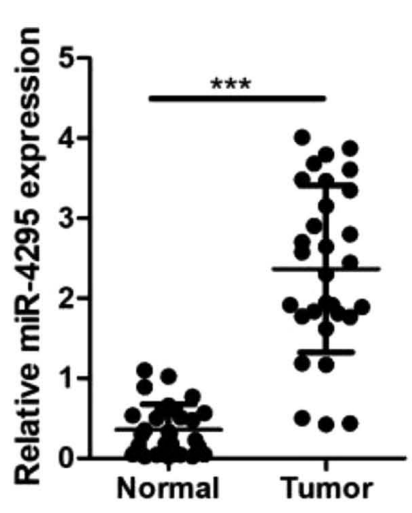

B

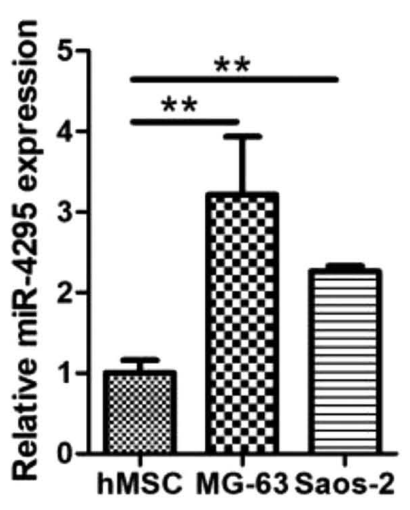

C

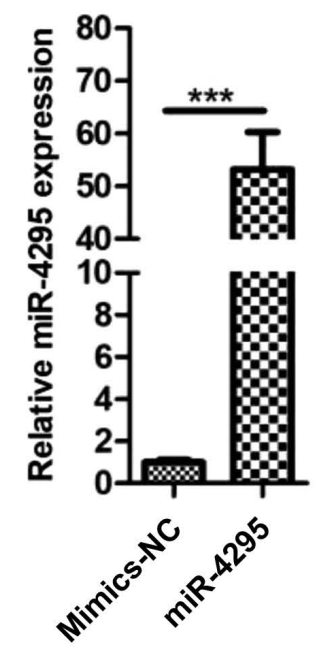

Figure 1. Expression levels of miR-4295 mRNA are significantly increased in OS tissues and cells. (A) Expression levels of miR-4295 in 30 samples of OS and adjacent normal tissues analyzed using reverse transcription PCR. Statistical analysis was evaluated using a paired Student's t-test. (B) Expression levels of miR-4295 in MG-63 and Saos-2 cell lines. Statistical analysis was evaluated using one-way analysis of variance and Tukey's post hoc test. (C) Expression levels of miR-4295 in the MG-63 cell line after transfection of miR-4295 mimics. Statistical analysis was evaluated using two-sided independent Student's t-test. ${ }^{* *} \mathrm{P}<0.01,{ }^{* * *} \mathrm{P}<0.001$. miR, microRNA; OS, osteosarcoma.

captured immediately and again at 24-h after scrapping using light microscopy (magnification, x100).

Dual luciferase assay. A dual luciferase assay was used to detect the direct binding of miR-4295 to the target gene IRF1. The binding sites of the IRF1 3' untranslated region (UTR) and miR-4295 were identified using the RNAhybrid database (http://alk.ibms.sinica.edu. tw/cgi-bin/RNAhybrid/RNAhybrid.cgi). Segmented 3'UTRs of the IRFI gene were amplified. After sequencing and blast validation, these fragments were inserted into the PMIRGLO (Addgene, Inc.) vector to construct recombinant plasmids. The firefly luciferase gene contained in the vector was used as a reference for normalization. A total of $5 \times 10^{4} \mathrm{MG}-63 /$ Saos- 2 cells per well were seeded into 12-well dishes and transfected with IRF1 luciferase reporter plasmids (100 ng) using Lipofectamine 2000. For overexpression and inhibition of miR-4295 experiments, 200 pmol mimics/inhibitor or miRNA NC were co-transfected along with plasmids, and luciferase activity was measured 48-h later. To measure the luciferase activity, cells were lysed in $1 \mathrm{x}$ lysis buffer provided with the Dual-Luciferase ${ }^{\circledR}$ Reporter Assay system (Promega Corporation) and luminescence was measured by adding luciferase assay reagent as per the manufacturer's protocol. Luciferase activity was measured using the Dual-Luciferase ${ }^{\circledR}$ Reporter Assay system (Promega Corporation). The assays were independently repeated $\geq$ three times.

Statistical analysis. Statistical analysis was performed using SPSS software version 13.0 (SPSS, Inc.). All experiments were performed in triplicate. The data shown are the mean \pm SD. Multiple group comparisons were analyzed using one-way analysis of variance and Tukey's post hoc test, with virus infection treatment as the betweenvsubjects factor. Paired Student's t-test was used to analyze the significance of mRNA levels in OS and adjacent normal tissues. Unpaired Student's t-test was used to analyze the significance of cell proliferation assay and the Transwell migration/invasion assay. A paired t-test was used to analyze the relative miR-4295 expression levels between paired normal and tumor tissues. Correlation analysis was evaluated using Pearson's correlation. All comparisons were two-tailed. $\mathrm{P}<0.05$ was considered to indicate a statistically significant difference.

\section{Results}

Levels of miR-4295 are significantly increased in OS tissues and cells. To investigate the potential function of miR-4295 in OS, the expression levels of miR-4295 in 30 samples of OS and adjacent normal tissues were detected using RT-qPCR. As shown in the Fig. 1A, miR-4295 expression was significantly increased in OS tissues compared with the paired normal tissues $(\mathrm{P}<0.01)$. Moreover, the expression levels of miR-4295 in OS cell lines (MG-63 and Saos-2) were significantly higher compared with those in the normal hMSC cells (Fig. 1B). The data indicated that miR-4295 expression may be associated with OS progression. As shown in the Fig. 1C, miR-4295 expression was significantly increased after transfection with miR-4295 mimics compared with the NC.

miR-4295 promotes OS cell proliferation. To explore the effects of miR-4295 on OS cells in vitro, miR-4295 mimics/mimics-NC, miR-4295 inhibitor/inhibitor-NC were transfected into MG-63 and Saos-2 cells. Upregulated miR-4295 expression significantly increased the proliferation ability of OS cells. In contrast, miR-4295-knockdown decreased MG-63 and Saos-2 cells proliferation ability (Fig. 2A and B, respectively). These data showed that miR-4295 promotes OS cell proliferation.

miR-4295 promotes the migration and invasion of OS cells. A Transwell and wound-healing assay were carried out to 
A

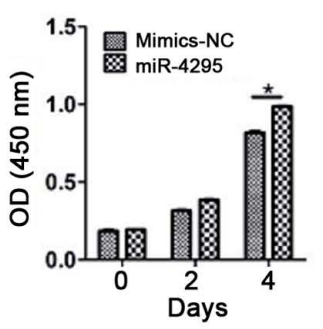

MG-63

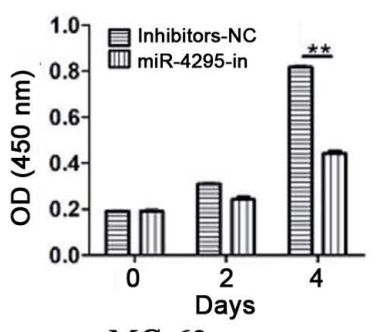

B

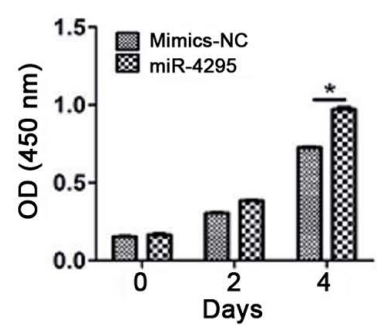

Saos-2

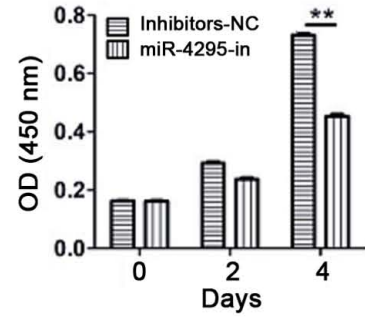

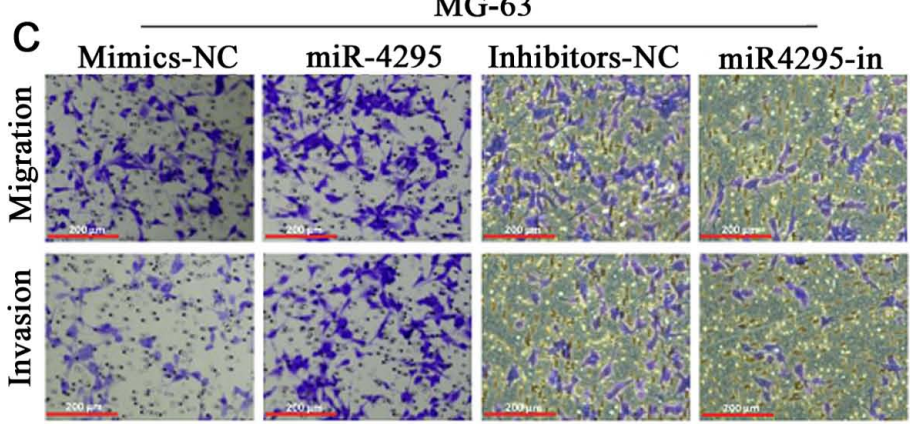

E Migration

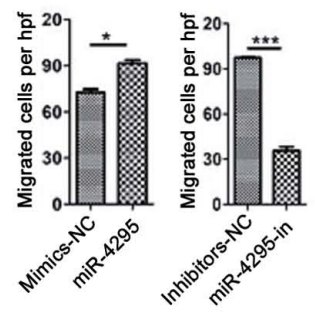

Invasion

Saos-2
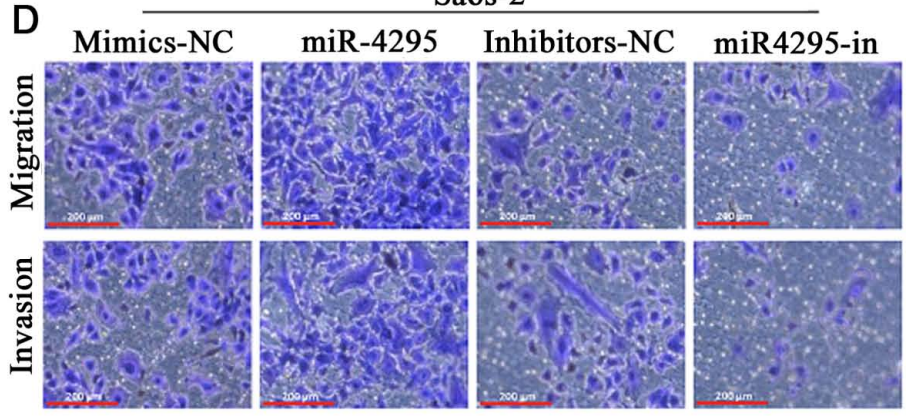

F

Migration

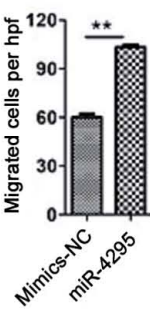

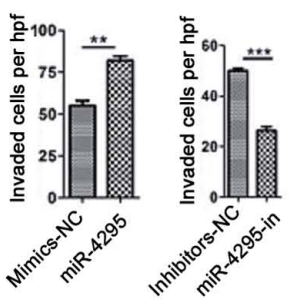

G
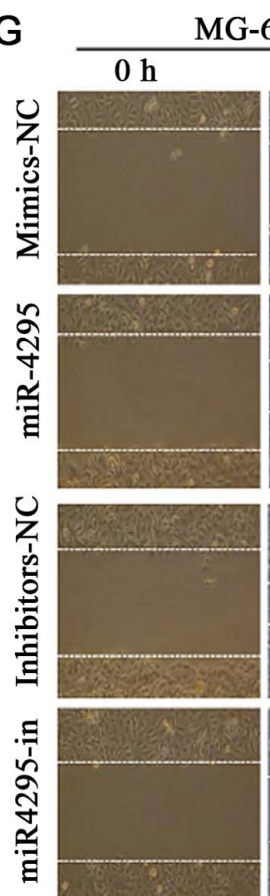

$24 \mathrm{~h}$
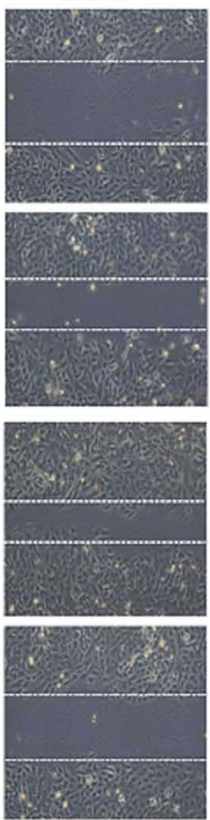

$\mathrm{H}$
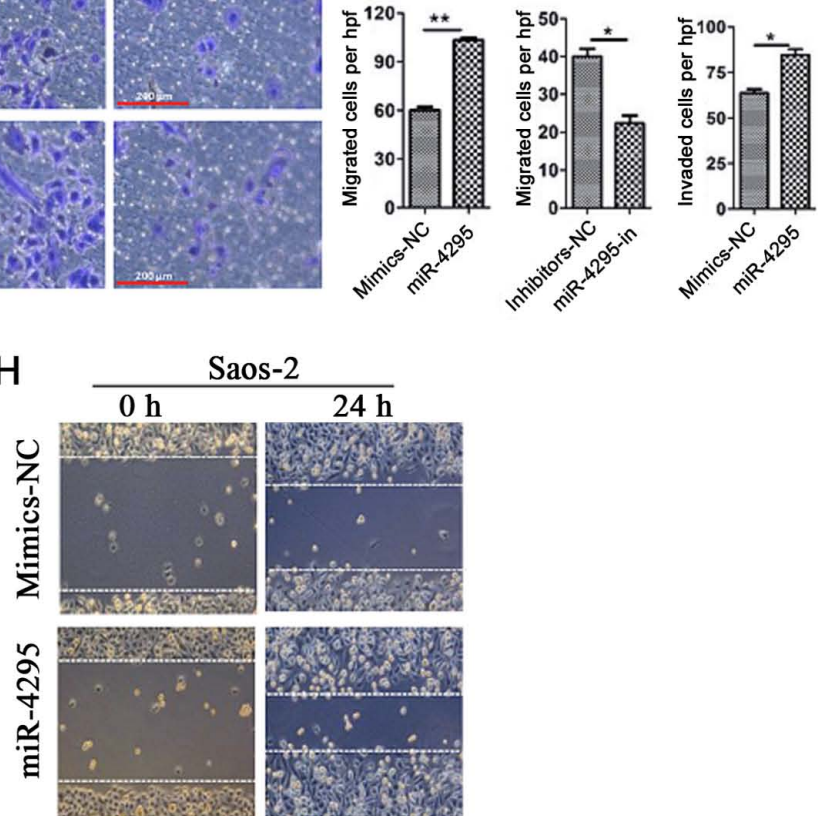

Invasion

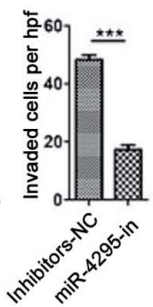

Figure 2. Effects of miR-4295 on the proliferation, migration and invasion of OS cells. (A and B) Cell Counting Kit-8 assay of MG-63 and Saos-2 cells after transfection with miR-4295 mimics/mimics-NC and miR-4295 inhibitor/inhibitor-NC. Statistical analysis was evaluated using two-sided independent Student's t-tests. (C and D) Migration and invasion of MG-63 and Saos-2 cells after transfection with miR-4295 mimics/mimics-NC and miR-4295 inhibitor/inhibitor-NC. (E and F) Three fields of the indicated cells were evaluated. Means + SD are shown from three independent experiments performed in triplicate Statistical analysis was performed using two-sided independent Student's t-tests. (G and H) The wound-healing assay of MG-63 and Saos-2 cells after transfected with miR-4295 mimics/mimics-NC, miR-4295 inhibitor/inhibitor-NC, respectively. Scale bar, $200 \mu \mathrm{m} .{ }^{*} \mathrm{P}<0.05,{ }^{* *} \mathrm{P}<0.01,{ }^{* * * *} \mathrm{P}<0.001 . \mathrm{miR}$, microRNA; OS, osteosarcoma; NC, negative control. 
A

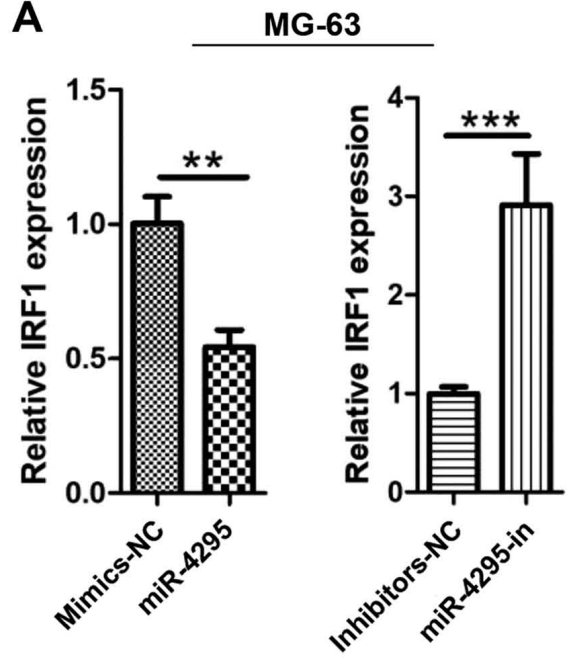

B

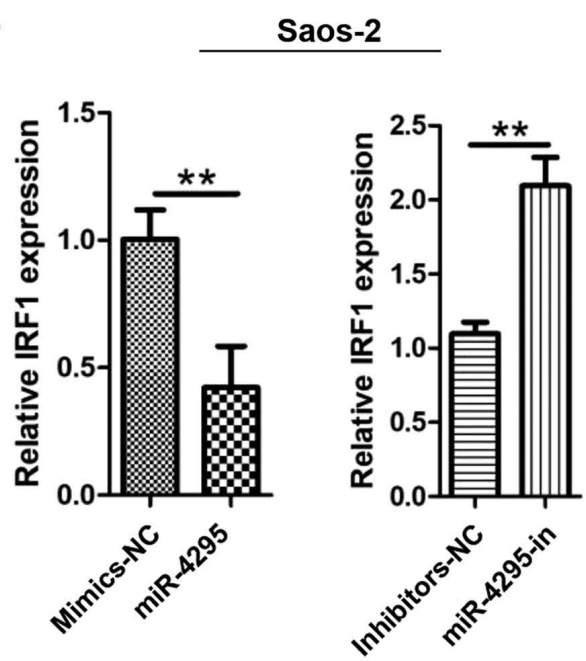

IRF1-3'UTR 5 '...UCCCAGGGCUGGCUCUGCACUAA.. miR-4295 $\quad 3^{\prime} \quad$ UUCCUUUUGUAACGUGAC

C

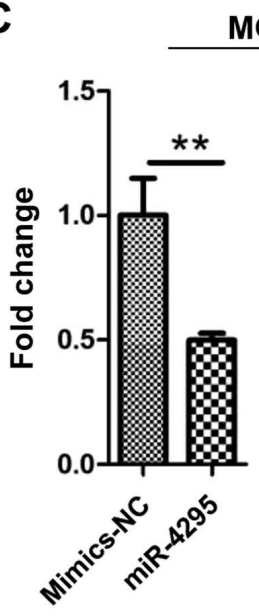

MG-63

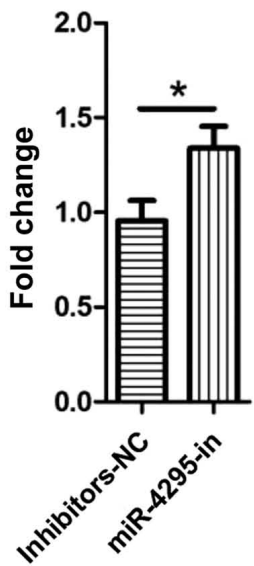

D

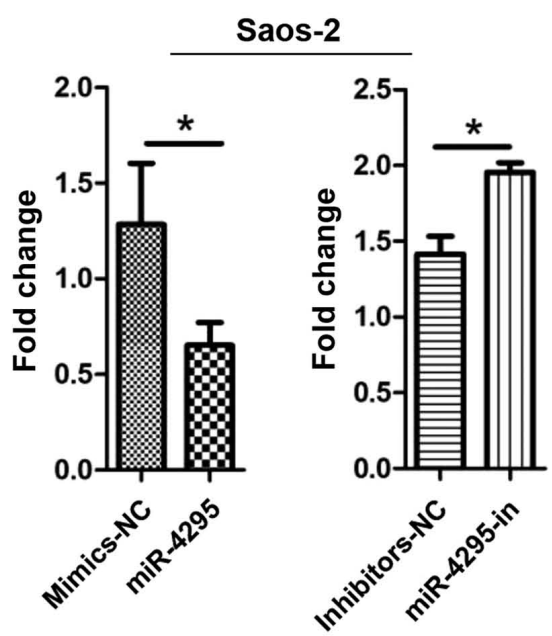

Figure 3. miR-4295 targets the IRF1 gene directly. (A and B) Expression levels of IRF1 after upregulation or knockdown of miR-4295 in MG-63 and Saos-2 cells. (C and D) Luciferase activity of the PMIRGLO-IRF1 reporter in MG-63 and Saos-2 cells was identified after co-transfection of the IRF1 3'UTR plasmid with miR-4295 mimics/mimics-NC or miR-4295 inhibitor/inhibitor-NC. Statistical analysis was evaluated using independent Student's t test. * $<<0.05$, ${ }^{* * *} \mathrm{P}<0.01,{ }^{* * *} \mathrm{P}<0.001$. IRF1, interferon regulatory factor 1 ; miR, microRNA; NC, negative control; UTR, untranslated region. Scale bar, $200 \mu \mathrm{m}$.

determine the influence of miR-4295 on the cell migration and invasion in vitro. It was demonstrated that MG-63 and Saos-2 cells transfected with miR-4295 mimics had increased migration and invasion compared with cells transfected with miR-NC (Fig. 2C-F), while cells treated with miR-4295 inhibitor exhibited a significant decrease in cell migration and invasion compared with cells treated with inhibitor-NC (Fig. 2C-F). Taken together, these results showed that overexpression of miR-4295 facilitated the migration and invasion of OS cells.

miR-4295 influences $O S$ cell proliferation, migration and invasion by targeting the IRF1 gene. To further elucidate the underlying mechanisms of miR-4295-influenced cell proliferation, migration and invasion, the downstream signaling pathways of miR-4295 were analyzed. The Target Scan Human7.1 (http://www.targetscan.org/vert_71/) database was applied to predict target genes of miR-4295.IRF1, one of the top
20 candidate target genes, as our previous study was associated with IRF1 (data are not shown). The mRNA expression levels of $I R F 1$ were examined after overexpression or knockdown of miR-4295 in OS cells. The mRNA expression levels of IRFI were significantly suppressed when miR-4295 expression was upregulated, while IRFI expression increased when miR-4295 was knocked down in OS cells (Fig. 3A and B). To determine whether miR-4295 directly targeted the IRFI gene, the IRFI 3'UTR was predicted through using Target Scan Human7.1 online tool and cloned into PMIRGLO vector. Dual luciferase reporter assays were performed to assess the interaction between miR-4295 and IRF1. As shown in Fig. 3C and D, there was a significant downward trend of the luciferase activity in the co-transfection of the IRF1 3'UTR plasmid with miR-4295 mimic compared with the mimics-NC. Furthermore, the luciferase activity of PMIRGLO-IRF1 reporter in OS cells was found to be decreased by compared with the miR-4295 inhibitor-NC. These data suggested that miR-4295 could 


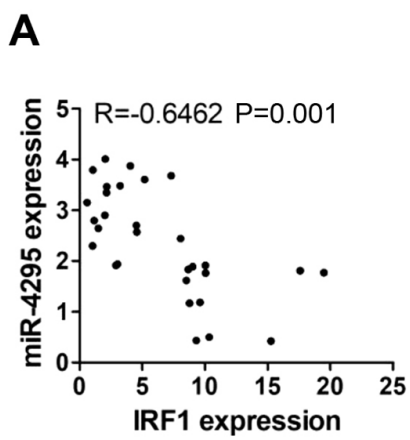

D LV-vector

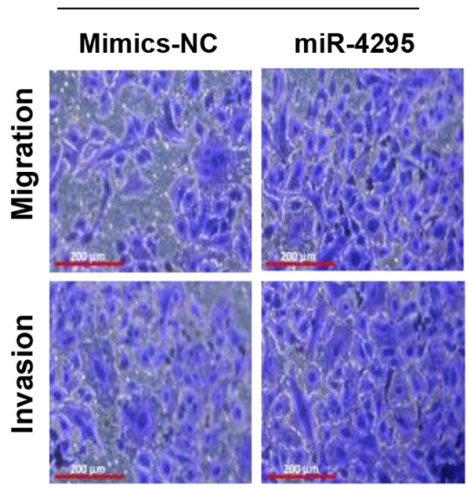

B

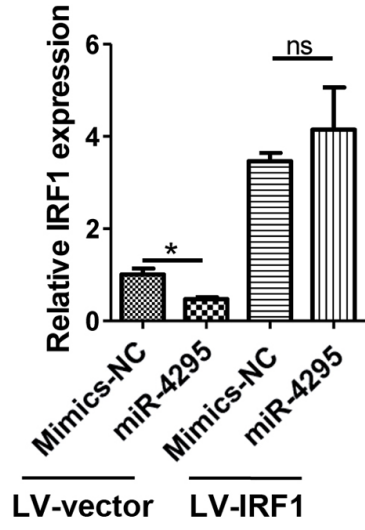

LV-IRF1

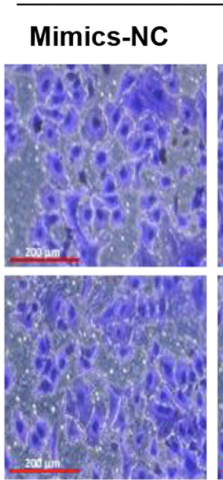

miR-4295

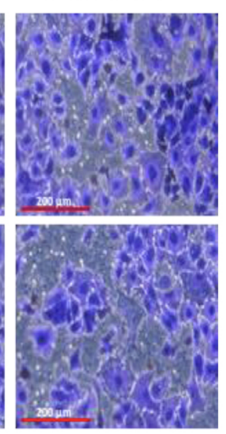

C

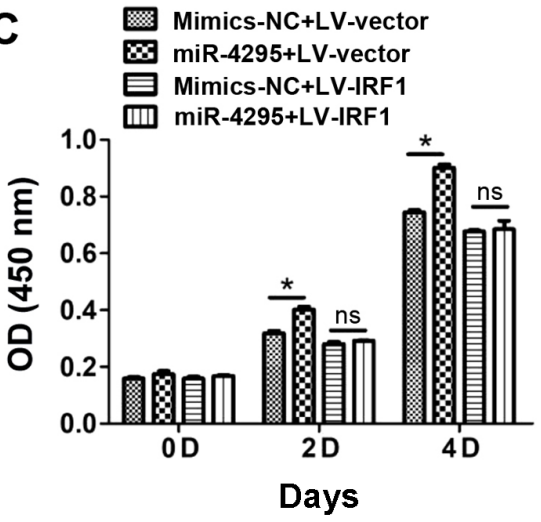

E

Migration

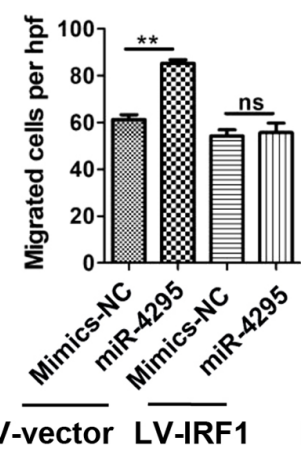

Invasion

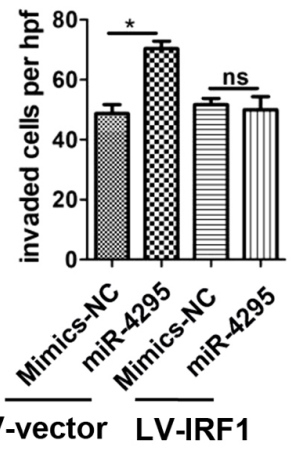

Figure 4. miR-4295 promotes Saos-2 cell migration and invasion by targeting the $I R F 1$ gene. (A) Correlation of expression levels of miR-4295 and $I R F 1$ in 30 samples of OS tissues analyzed using reverse transcription PCR. Statistical analysis was evaluated with the Pearson's correlation test. (B) Expression levels of IRF1 after transfection with LV-IRF1 and LV-NC lentivirus in Saos-2 cells transfected with mimics-NC or mir-4295 mimics. Statistical analysis was analyzed using one-way analysis of variance and Tukey's post hoc test. (C) Cell Counting Kit-8 assay of Saos-2 cells after transfection with LV-IRF1 and LV-NC lentivirus. Statistical analysis was analyzed using one-way analysis of variance and Tukey's post hoc test. (D) Migration and invasion of Saos-2 cells after transfection with LV-IRF1 and LV-NC lentivirus. (E) Three fields of the indicated cells were evaluated. Means + SD are shown from three independent experiments performed in duplicates. Statistical analysis was evaluated using one-way analysis of variance and Tukey's post hoc test. ${ }^{*} \mathrm{P}<0.05,{ }^{* *} \mathrm{P}<0.01$. miR, microRNA; IRF1, interferon regulatory factor 1; OS, osteosarcoma; NC, negative control; ns, not significant. Scale bar, $200 \mu \mathrm{m}$.

suppress the translation of IRF1 by targeting its 3'-UTR. Then, the mRNA expression levels of IRFI in $30 \mathrm{OS}$ tissues were detected, and the results indicated that the expression levels of miR-4295 were correlated with IRF1 (Fig. 4A).

To verify whether IRF1 could inhibit the miR-4295-mediated promotion of OS cell proliferation, migration and invasion, the LV-vector and LV-IRF1 lentivirus was used to transfect Saos-2 cells that were subsequently used in CCK-8 and Transwell assays (Fig. 4C-E). Cell proliferation, migration and invasion in miR-4295+LV-IRF1 group were similar to those in the mimics-NC+LV-IRF1 group. The results indicated that the miR-4295-mediated promotion of Saos-2 cell proliferation, migration and invasion was inhibited by transfection with LV-IRF1 lentivirus. These results suggested that miR-4295 promotes OS cell proliferation, migration and invasion by targeting IRFI gene.

\section{Discussion}

OS, the most common form of primary bone tumor, primarily affects children and adolescents (5). In the past few years, the prognosis of patients with OS had progressed very little, especially for patients with metastatic disease (26-28).
Despite encouraging progression in therapeutic management, the survival rates still remained unchanged over the past 40 years $(28,29)$. In recent years, with the development of genomics and the deepening of miRNA research, our understanding of the mechanisms underlying occurrence, progression and metastasis of OS have improved, especially in in terms of occurrence and progression $(30,31)$. It has been established that miRNAs play a key regulatory function in the progression of OS (31).

To explore the association of miRNAs with OS, bioinformatics techniques were used to screen for differentially expressed miRNAs in OS in the GEO database. In the GSE70367 database, it was demonstrated that miR-4295 expression was abnormally elevated in OS cell lines. Recent studies have found that miR-4925 might exert some function as a cancer-related miRNA in several types of cancer (20,31-33). It was reported that miR-4295 could inhibit glioma cell arrest at the $G_{0} / G_{1}$ phase, cause apoptosis and promote cell proliferation (19). Also, inhibition of miR-4295 suppressed cell proliferation and invasion through antagonizing the $\mathrm{Wnt} / \beta$-catenin signaling pathway in pancreatic ductal adenocarcinoma (20). In bladder cancer, overexpression of miR-4295 could promote cell proliferation, colony formation and migration, and downregulation of miR-4295 
induced cell cycle arrest and apoptosis (34). To characterize the potential role of miR-4295 in OS, the expression levels of miR-4295 were examined in 30 samples of OS and adjacent normal tissues in the present study. The results revealed that the expression of miR-4295 was significantly increased in OS tissues compared with the paired normal tissues. Moreover, the expression levels of miR-4295 in MG-63 and Saos-2 cell lines were significantly higher compared with in the normal hMSC cells. In addition, the functional assays showed that upregulation of miR-4295 could promote the proliferation, migration and invasion of OS cells. Taken together, these findings indicated that miR-4295 might act as a novel tumor activator in OS.

To elucidate the underlying molecular mechanisms of miR-4295, the downstream signaling pathways of miR-4295 were analyzed. Using the Target Scan Human7.1 database, the target genes of miR-4295 were predicted. The 20 top candidate target genes of miR-4295 were as follows: Lysine demethylase 2A (KDM2A), SLAIN motif family member 1 (SLAIN1), MDM4 regulator of P53 (MDM4), kruppel like factor 7 (KLF7), poly(A) specific ribonuclease Subunit PAN3 (PAN3), ectonucleotide pyrophosphatase/phosphodiesterase family member 5 (NPP5), F-Box protein 28 (FBXO28), MYB proto-oncogene like 1 (MYBL1), activin A receptor type 1 (ACVR1), cytoplasmic polyadenylation element binding protein 1 (CPEB1), carbohydrate sulfotransferase 1 (CHST1), phosphatidylinositol-4,5-bisphosphate 3-kinase catalytic subunit beta (PIK3CB), RNA 3'-terminal phosphate cyclase (RTCA), syntabulin (SYBU), interferon regulatory factor 1 (IRF1), ribosomal protein S6 kinase A5 (RPS6KA5), MAF BZIP transcription factor (MAF), dynein light chain LC8-type 2 (DYNLL2) and SET binding factor 2 (SBF2; data not shown). Our previous study showed that siRNA-mediated IRF1-knockdown could promote the proliferation and migration of Saos-2 cells (unpublished data). IRF1, a member of IRF family, could serve as a transcriptional regulator and tumor suppressor playing a crucial role in tumor cell growth and immune responses (35). Various studies have demonstrated that IRF1 can act as a tumor suppressor contributing several types of cancer (36). A recent study demonstrated that overexpression of IRF1 suppresses cell proliferation, migration and invasion, and blocked cell cycle progression of cholangiocarcinoma cells (36). As a tumor suppressor, upregulation of IRF-1 suppresses the transformed phenotype of ovarian cancer cells (37). IRF1 exerts its antiproliferative effect through repressing the transcription of a novel proliferation-related downstream target, the $K i-67$ gene, in a dose-dependent manner in renal carcinoma cells (38). Notably, upregulation of IRF-1 had been found to result in a 15-fold downregulation of survivin protein levels in breast carcinoma cells (39). The present study observed that the expression of $I R F I$ was suppressed by overexpression of miR-4295 and increased by knockdown of miR-4295 in OS cells. Meanwhile, the data demonstrated that the 3'-UTR region of IRFI had a conserved miR-4295 binding site. The 3'-UTRs of the IRFI gene were inserted into the pMIRGLO vector. The results of the luciferase assay confirmed that IRFI was a target gene of miR-4295 in OS cells. Meanwhile, the mutant 3'UTRs of the IRF1 gene were constructed but these were not successfully inserted into pMIRGLO vector. Experiments using such vectors could validate the present conclusion. Taken together, the results revealed that miR-4295 may act as a tumor activator by targeting IRFI during the progression of OS.

In conclusion, miR-4295 promoted proliferation, migration and invasion of OS cells by targeting the IRFI gene. The resolved functions of miR-4295 may provide novel insight into the mechanisms of OS metastasis, and inhibition of miR-4295 may have value as a therapeutic strategy for the treatment of OS.

\section{Acknowledgements}

Not applicable.

\section{Funding}

No funding was received.

\section{Availability of data and materials}

The data during the present study are available from the corresponding author on reasonable request.

\section{Authors' contributions}

ZZ provided the samples. JC and BH performed the experiments. JC, JD and KY analyzed the data. JC wrote the manuscript. JC and ZZ designed and supervised the study and wrote the manuscript. All authors read and approved the final manuscript.

\section{Ethics approval and consent to participate}

This study was approved by the Ethics Committee of Xiangyang No. 1 People's Hospital (Hubei, China). Written informed consent was obtained from each patient prior to participation.

\section{Patient consent for publication}

Not applicable.

\section{Competing interests}

The authors declare that they have no competing interests.

\section{References}

1. Clark JC, Dass CR and Choong PF: A review of clinical and molecular prognostic factors in osteosarcoma. J Cancer Res Clin Oncol 134: 281-297, 2008.

2. Bacci G, Briccoli A, Rocca M, Ferrari S, Donati D, Longhi A, Bertoni F, Bacchini P, Giacomini S, Forni C, et al: Neoadjuvant chemotherapy for osteosarcoma of the extremities with metastases at presentation: Recent experience at the Rizzoli Institute in 57 patients treated with cisplatin, doxorubicin, and a high dose of methotrexate and ifosfamide. Ann Oncol 14: 1126-1134, 2003.

3. Kimura K, Nakano T, Park YB, Tani M, Tsuda H, Beppu Y, Moriya $\mathrm{H}$ and Yokota J: Establishment of human osteosarcoma cell lines with high metastatic potential to lungs and their utilities for therapeutic studies on metastatic osteosarcoma. Clin Exp Metastasis 19: 477-485, 2002.

4. Morishita T, Mii Y, Miyauchi Y, Miura S, Honoki K, Aoki M, Kido A, Tamai S, Tsutsumi M and Konishi Y: Efficacy of CDDP and AGM-1470 chemotherapy against lung metastasis in rat osteosarcoma depends on the timing of combined administration. Jpn J Clin Oncol 27: 236-239, 1997. 
5. Whelan J, Seddon B and Perisoglou M: Management of osteosarcoma. Curr Treat Options Oncol 7: 444-455, 2006.

6. Sampson VB, Yoo S, Kumar A, Vetter NS and Kolb EA: MicroRNAs and potential targets in osteosarcoma: Review. Front Pediatr 3: 69, 2015.

7. Zhang Y, Meng W and Cui H: LncRNA CBR3-AS1 predicts unfavorable prognosis and promotes tumorigenesis in osteosarcoma. Biomed Pharmacother 102: 169-174, 2018.

8. Wu D, Nie X, Ma C, Liu X, Liang X, An Y, Zhao B and Wu X: RSF1 functions as an oncogene in osteosarcoma and is regulated by XIST/miR-193a-3p axis. Biomed Pharmacother 95: 207-214, 2017.

9. Cheng DD, Li SJ, Zhu B, Zhou SM and Yang QC: EEF1D overexpression promotes osteosarcoma cell proliferation by facilitating Akt-mTOR and Akt-bad signaling. J Exp Clin Cancer Res 37: 50, 2018

10. Bartel DP: MicroRNAs: Target recognition and regulatory functions. Cell 136: 215-233, 2009.

11. Lee RC, Feinbaum RL and Ambros V: The C. elegans heterochronic gene lin-4 encodes small RNAs with antisense complementarity to lin-14. Cell 75: 843-854, 1993.

12. Treiber T, Treiber N and Meister G: Regulation of microRNA biogenesis and its crosstalk with other cellular pathways. Nat Rev Mol Cell Biol 20: 5-20, 2019.

13. Lujambio A and Lowe SW: The microcosmos of cancer. Nature 482: 347-355, 2012.

14. Zhang C: Novel functions for small RNA molecules. Curr Opin Mol Ther 11: 641-651, 2009.

15. Li Y, Liu J, Liu ZZ and Wei WB: MicroRNA-145 inhibits tumour growth and metastasis in osteosarcoma by targeting cyclin-dependent kinase, CDK6. Eur Rev Med Pharmacol Sci 20: 5117-5125, 2016.

16. Wang X, Lin Y, Peng L, Sun R, Gong X, Du J and Zhang X: MicroRNA-103 promotes proliferation and inhibits apoptosis in spinal osteosarcoma cells by targeting p57. Oncol Res 26 933-940, 2018

17. Nan YH, Wang J, Wang Y, Sun PH, Han YP, Fan L, Wang KC, Shen FJ and Wang WH: MiR-4295 promotes cell growth in bladder cancer by targeting BTG1. Am J Transl Res 8: 4892-4901, 2016.

18. Zhao F, Yang X, Xu G, Bi J, Lv R and Huo R: Propranolol suppresses HUVEC viability, migration, VEGF expression and promotes apoptosis by downregulation of miR-4295. J Cell Biochem 120: 6614-6623, 2019.

19. Li X,Zheng J, Diao H and Liu Y: RUNX3 is down-regulated in glioma by Myc-regulated miR-4295. J Cell Mol Med 20: 518-525, 2016.

20. Yuan Q, Zhang Y, Li J, Cao G and Yang W: High expression of microRNA-4295 contributes to cell proliferation and invasion of pancreatic ductal adenocarcinoma by the down-regulation of Glypican-5. Biochem Biophys Res Commun 497: 73-79, 2018.

21. Ivashkiv LB and Donlin LT: Regulation of type I interferon responses. Nat Rev Immunol 14: 36-49, 2014.

22. Eckhardt I, Weigert A and Fulda S: Identification of IRF1 as critical dual regulator of Smac mimetic-induced apoptosis and inflammatory cytokine response. Cell Death Dis 5: e1562, 2014.

23. Alessandro R and Nicole MC: Genome-wide Identification of IRF1 binding sites reveals extensive occupancy at cell death associated genes. J Carcinog Mutagen: S6-S009, 2013.

24. Armstrong MJ, Stang MT, Liu Y, Gao J, Ren B, Zuckerbraun BS, Mahidhara RS, Xing Q, Pizzoferrato E and Yim JH: Interferon regulatory factor 1 (IRF-1) induces p21WAF1/CIP1 dependent cell cycle arrest and p21WAF1/CIP1 independent modulation of survivin in cancer cells. Cancer Lett 319: 56-65, 2012.
25. Livak KJ and Schmittgen TD: Analysis of relative gene expression data using real-time quantitative PCR and the 2(-Delta Delta C(T)) method. Methods 25: 402-408, 2001.

26. Misaghi A, Goldin A, Awad M and Kulidjian AA: Osteosarcoma: A comprehensive review. SICOT J 4: 12, 2018

27. Li S, Zhang T, Xu W, Ding J, Yin F, Xu J, Sun W, Wang H, Sun M, Cai $Z$ and Hua Y: Sarcoma-targeting peptide-decorated polypeptide nanogel intracellularly delivers shikonin for upregulated osteosarcoma necroptosis and diminished pulmonary metastasis. Theranostics 8: 1361-1375, 2018.

28. Xu J, Wang $\mathrm{H}, \mathrm{Hu}$ Y, Zhang YS, Wen L, Yin F, Wang Z, Zhang Y, Li S, Miao Y, et al: Inhibition of CaMKII $\alpha$ activity enhances antitumor effect of fullerene C60 nanocrystals by suppression of autophagic degradation. Adv Sci (Weinh) 6: 1801233, 2019

29. Collins M, Wilhelm M, Conyers R, Herschtal A, Whelan J, Bielack S, Kager L, Kühne T, Sydes M, Gelderblom H, et al: Benefits and adverse events in younger versus older patients receiving neoadjuvant chemotherapy for osteosarcoma: Findings from a meta-analysis. J Clin Oncol 31: 2303-2312, 2013.

30. Kushlinskii NE, Fridman MV and Braga EA: Molecular mechanisms and microRNAs in osteosarcoma pathogenesis. Biochemistry (Mosc) 81: 315-328, 2016

31. Hutanu D, Popescu R, Stefanescu H, Pirtea L, Candea A, Sarau C, Boruga O, Mehdi L, Ciuca I and Tanasescu S: The molecular genetic expression as a novel biomarker in the evaluation and monitoring of patients with osteosarcoma-subtype bone cancer disease. Biochem Genet 55: 291-299, 2017.

32. Huang G, Nishimoto K, Yang Y and Kleinerman ES: Participation of the Fas/FasL signaling pathway and the lung microenvironment in the development of osteosarcoma lung metastases. Adv Exp Med Biol 804: 203-217, 2014.

33. Liu X and Zhang Y: Lycium barbarum polysaccharides alleviate hydrogen peroxide-induced injury by up-regulation of miR-4295 in human trabecular meshwork cells. Exp Mol Pathol 106 109-115, 2019.

34. Jin H, Xu J, Guo X, Huang H, Li J, Peng M, Zhu J, Tian Z, Wu XR, Tang MS and Huang C: XIAP RING domain mediates miR-4295 expression and subsequently inhibiting p63 $\alpha$ protein translation and promoting transformation of bladder epithelial cells. Oncotarget 7: 56540-56557, 2016.

35. Thygesen SJ and Stacey KJ: IRF1 and IRF2 regulate the non-canonical inflammasome. EMBO Rep 20: e48891, 2019.

36. Wan P, Zhang J, Du Q and Geller DA: The clinical significance and biological function of interferon regulatory factor 1 in cholangiocarcinoma. Biomed Pharmacother 97: 771-777, 2018.

37. Pavan S, Olivero M, Corà D and Di Renzo MF: IRF-1 expression is induced by cisplatin in ovarian cancer cells and limits drug effectiveness. Eur J Cancer 49: 964-973, 2013.

38. Lorenzi S, Forloni M, Cifaldi L, Antonucci C, Citti A, Boldrini R, Pezzullo M, Castellano A, Russo V, van der Bruggen P, et al: IRF1 and NF-kB restore MHC class I-restricted tumor antigen processing and presentation to cytotoxic $\mathrm{T}$ cells in aggressive neuroblastoma. PLoS One 7: e46928, 2012.

39. Pizzoferrato E, Liu Y, Gambotto A, Armstrong MJ, Stang MT, Gooding WE, Alber SM, Shand SH, Watkins SC, Storkus WJ and Yim JH: Ectopic expression of interferon regulatory factor-1 promotes human breast cancer cell death and results in reduced expression of survivin. Cancer Res 64: 8381-8388, 2004. 